\title{
Mixed finite element and atomistic formulation for complex crystals
}

\section{Citation}

Tadmor, E. B., G. S. Smith, N. Bernstein, and E. Kaxiras. 1999. "Mixed Finite Element and Atomistic Formulation for Complex Crystals." Physical Review B 59 (1): 235-45. https:// doi.org/10.1103/physrevb.59.235.

\section{Permanent link}

http://nrs.harvard.edu/urn-3:HUL.InstRepos:41384078

\section{Terms of Use}

This article was downloaded from Harvard University's DASH repository, and is made available under the terms and conditions applicable to Other Posted Material, as set forth at http:// nrs.harvard.edu/urn-3:HUL.InstRepos:dash.current.terms-of-use\#LAA

\section{Share Your Story}

The Harvard community has made this article openly available.

Please share how this access benefits you. Submit a story.

Accessibility 


\title{
Mixed finite element and atomistic formulation for complex crystals
}

\author{
E. B. Tadmor, ${ }^{*}$ G. S. Smith, N. Bernstein,$^{\dagger}$ and E. Kaxiras \\ Division of Engineering and Applied Science, Harvard University, Cambridge, Massachusetts 02138
}

(Received 21 August 1998)

\begin{abstract}
A general formulation for the analysis of complex Bravais crystals using atomic energy functionals embedded within a finite element framework is presented. The method uses atomistic potentials to determine the constitutive response of the system. Unlike traditional finite element methods, the nonlinear elastic effects, the symmetries of the underlying crystal, and the possibility of uniform structural phase transformations are naturally included in this formulation. Explicit expressions for empirical energy functionals with separable two- and three-body potentials, and semiempirical tight-binding energy functionals with two-center integrals are presented. A simple application to silicon underscores the importance of including internal relaxation in a finite element treatment of a complex crystal. In a forthcoming companion paper, the method presented here is applied to the nanoindentation of silicon. [S0163-1829(99)14301-7]
\end{abstract}

\section{INTRODUCTION}

Computer simulations are a valuable tool for understanding the fundamental processes that determine how a material responds to external loads. Broadly speaking, there are two classes of approaches that have proven particularly useful: atomistic and continuum simulations. Atomistic approaches provide detailed information about microscopic processes, and advances in computer design and algorithms have made possible the simulation of ever larger systems. However, even the most ambitious atomic simulations using empirical potentials can only handle systems sizes of order $10^{9}$ atoms, which means that system dimensions generally cannot exceed a few hundred nanometers. Moreover, reliable results for large systems depend on the availability of high-quality, efficient energy functionals. Accurate, transferable empirical potentials are available for many pure metals and a few single-element covalent systems, but very few systems that contain more than one element. An alternative is to use quantum-mechanical energy functionals based on tightbinding or density-functional theory formulations. These are more transferable and can be applied to more complicated crystals, but are several orders of magnitude slower in computational speed than empirical potentials.

Continuum mechanics simulations have a different set of strengths. Since atomic details are coarse grained out of the formulation, much larger systems can be treated. But the constitutive equations that relate the strain and stress in a system are often empirically determined, making their reliability over a wide range of deformations dubious. In addition to this difficulty, the lack of an explicit connection between the continuum fields and atomic degrees of freedom makes it difficult to take into account the important properties of a crystal that depend on its atomic structure, such as crystal symmetries, invariance of a primitive cell with respect to shear by a full Burger's vector, and for complex crystals, the possibility of structural phase transformations. We seek to overcome these deficiencies in traditional continuum approaches by explicitly embedding atomistic totalenergy calculations within a finite element framework.

We focus on a recently introduced approach referred to as the quasicontinuum method. ${ }^{1}$ In this approach a continuum finite element formulation is used to characterize the mechanical response of a given system. The difference from standard finite element methodologies lies in that the constitutive response of the system is obtained from an atomistic calculation rather than an empirical phenomenological rule. The basic idea is that every point in a continuum corresponds to a very large region on the atomic scale. Thus, the constitutive response at that point, i.e., the stress-strain relation there, may be obtained by deforming the underlying crystal structure by the local strain to obtain the local state of stress. This approximation will be adequate as long as the variation of the continuum fields is "slow" on the atomic scale. When this breaks down, such as near defect cores, the quasicontinuum method includes a nonlocal limit where the inhomogeneous deformation is specifically accounted for. Here, however, we focus on the local limit of the quasicontinuum formulation, leaving the treatment of the nonlocal defect effects to a future publication.

In its original version the quasicontinuum method was formulated with simple Bravais crystals in mind. Many useful materials (silicon being one example) have more than one atom per primitive unit cell. Furthermore, with a sufficiently large unit cell, a realistic representation of amorphous structures and alloys could be modeled, permitting finite element simulations of these complex materials. Extending the quasicontinuum method to simulate materials with multiple atoms in a unit cell is the focus of this paper. We present a formulation that maintains the advantages of traditional finite element methods, including correct treatment of the far-field boundary conditions. At the same time, the formulation naturally incorporates the anisotropy and symmetries of the material, all the nonlinearities associated with finite deformation, and the possible occurrence of structural phase transformations. Finally, the efficiency of the finite element formulation permits computationally expensive quantummechanical energy functionals to be applied to significantly larger systems than would be possible in atomistic simulations.

This generalized quasicontinuum method is ideal for simulating a variety of complex systems. In a forthcoming 
companion paper, the method is applied to nanoindentation of silicon. ${ }^{2}$ Additional applications include modeling the constitutive response of ferroelectric materials, ${ }^{3}$ simulating microelectromechanical systems devices, and modeling highly strained nickel-aluminum alloys. All these systems have multiple atoms in a unit cell, and internal relaxation plays an important role in determining the response of the system to external loads. Furthermore, in some systems large deformation can lead to phase transformations: silicon has been observed to transform into a conducting phase under an indenter, ${ }^{4}$ and $\mathrm{NiAl}$ can undergo martensitic transformations near a crack tip. ${ }^{5}$ The formulation of the quasicontinuum method presented here naturally captures these diverse physical phenomena.

The outline of the paper is as follows: Section II briefly describes the finite element framework. Section III focuses on the atomistic aspects of the formulation. First, the general expressions for the stresses and elastic constants for a general complex crystal are derived. Second, the explicit energy density expressions that link the continuum and atomic descriptions are presented for the case of a separable two- and three-body potential, and for the case of a two-center tightbinding Hamiltonian. Section IV shows how internal relaxation dramatically affects the behavior of system undergoing simple shear.

\section{FINITE DEFORMATION CONTINUUM FRAMEWORK}

The continuum formulation adopted here is that of finite deformation. Thus, no assumptions are made regarding the smallness of the strains. This can be important in microsystems where large mismatch and thermal strains as well as large mechanical operating strains are sometimes encountered. Within a finite deformation framework we differentiate between a solid in its reference or undistorted state (also referred to as the material frame) and its current or deformed state (called the spatial frame). Consider a crystal occupying a configuration $B_{0}$ in the reference frame which is mapped to its current shape $B$ by the deformation mapping $\phi(\mathbf{X})$ (see Fig. 1). Thus every point $\mathbf{X}$ in the reference configuration is mapped to some point $\mathbf{x}$ in the current configuration by

$$
\mathbf{x}=\phi(\mathbf{X})=\mathbf{X}+\mathbf{u}(\mathbf{X}),
$$

where $\mathbf{u}(\mathbf{X})$ is the displacement at point $\mathbf{X}$. The deformation of an infinitesimal neighborhood of point $\mathbf{X}$ (represented as a shaded circle in the figure) is completely characterized by the linear part of $\phi(\mathbf{X})$. This defines an affine mapping,

$$
d \mathbf{x}=\mathbf{F} d \mathbf{X},
$$

where $\mathbf{F}$ is the deformation gradient,

$$
\mathbf{F}=\nabla_{0} \phi=\frac{\partial \phi}{\partial \mathbf{X}}=\mathbf{I}+\frac{\partial \mathbf{u}}{\partial \mathbf{X}},
$$

where $\mathbf{I}$ is the identity tensor. In indicial notation, $F_{i J}$ $=\phi_{i, J}$ and $d x_{i}=F_{i J} d X_{J}$ where upper case indices refer to the material frame, lower case indices to the spatial frame, $(\cdot)_{J}$ indicates differentiation with respect to $X_{J}$ and Einstein's summation convention on repeating indices is observed.

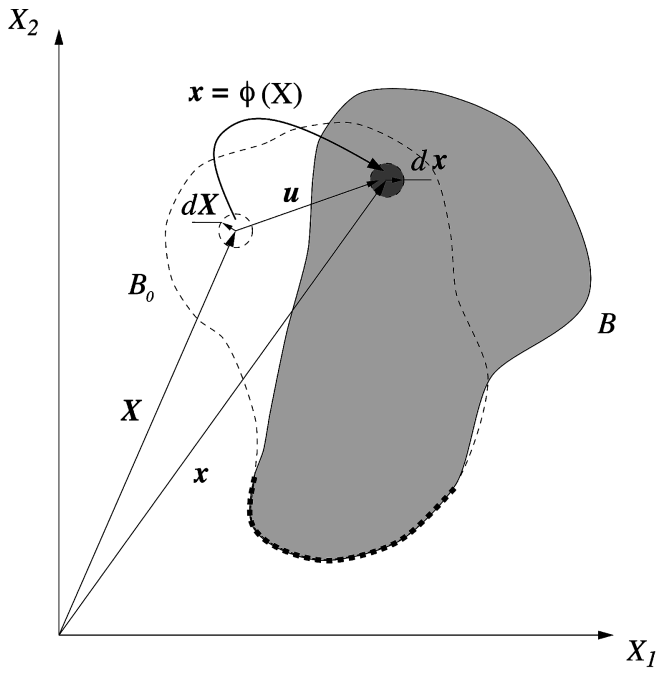

FIG. 1 . The deformation mapping relating the material $\left(B_{0}\right)$ and spatial $(B)$ configurations. Displacements are specified on the dark dashed part of the boundary $\left(\partial B_{0 U}\right)$ and tractions on the remaining boundary $\left(\partial B_{0 T}\right)$.

The deformation mapping and its gradient characterize the kinematics of the deformation. The constitutive nature of the material is introduced through the strain energy density function $W$ which relates the energy at a point to the local state of deformation there. From the hypothesis of locality and use of the entropy production inequality it may be shown ${ }^{6}$ that $W$ can only be a function of $\mathbf{F}$, i.e., $W=W(\mathbf{F})$ and not its derivatives or any other variables. Furthermore, by invoking the postulate of material frame indifference it can also be shown ${ }^{6}$ that the dependence of $W$ on $F$ can only be through the right Cauchy-Green tensor $\mathbf{C}=\mathbf{F}^{T} \mathbf{F}$. However, for the current formulation it is more convenient to work in terms of $\mathbf{F}$.

We may now formulate the general boundary value problem. To this end, we partition the reference boundary $\partial B_{0}$ into a displacement component $\partial B_{0 U}$ and a traction component $\partial B_{0 T}$ (see Fig. 1). The solid is subjected to prescribed displacements $\overline{\mathbf{u}}$ on $\partial B_{0 U}$ and prescribed tractions $\overline{\mathbf{T}}$ on $\partial B_{0 T}$. Stable configurations of the crystal are identified with minimizers of the potential energy,

$$
\Pi=\inf _{\mathbf{u}}\left\{\int_{B_{0}} W(\mathbf{F}) d V_{0}-\int_{\partial B_{0 T}}(\overline{\mathbf{T}} \cdot \mathbf{u}) d S_{0}\right\},
$$

where $\mathbf{F}$ is a function of $\mathbf{u}$ through Eq. (3), and the trial deformations $\mathbf{u}$ satisfy the essential boundary condition $\mathbf{u}$ $=\overline{\mathbf{u}}$ on $\partial B_{0 U}$.

To solve the problem we discretize our continuum into a set of finite elements bounded by nodes (see Fig. 2). The continuous displacement field $\mathbf{u}$ is now approximated by finite element interpolation from its nodal values $\mathbf{u}_{a}$,

$$
\mathbf{u}(\mathbf{X}) \simeq \widetilde{\mathbf{u}}(\mathbf{X})=\sum_{a=1}^{N_{n}} \mathbf{u}_{a} N_{a}(\mathbf{X})
$$

where there are $N_{n}$ nodes in the mesh and $N_{a}(\mathbf{X})$ are the standard finite element interpolation, or "shape" functions. The potential energy in Eq. (4) may also be rewritten in the discretized form 


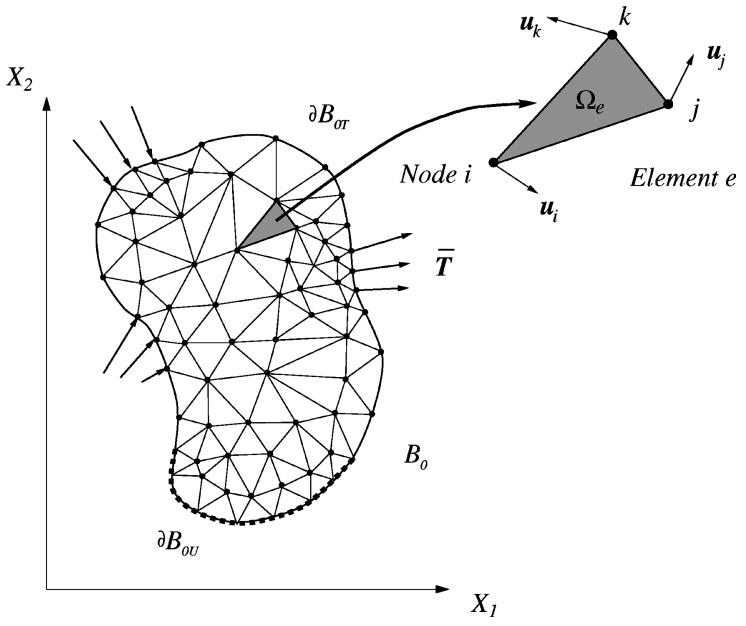

FIG. 2. Finite element mesh of reference solid $B_{0}$ showing boundary conditions and nodal degrees of freedom.

$$
\widetilde{\Pi}\left(\left\{\mathbf{u}_{i}\right\}\right)=\sum_{e=1}^{N_{e}} \int_{\Omega_{e}} W(\mathbf{F}) d V_{0}-\sum_{a=1}^{N_{n}} \int_{\partial B_{0 T}}\left(\overline{\mathbf{T}} \cdot \mathbf{u}_{a}\right) N_{a} d S_{0},
$$

where $N_{e}$ is the number of elements and $\Omega_{e}$ is the domain of element $e$. All integrals in Eq. (6) are normally carried out by numerical quadrature, ${ }^{7}$ e.g.,

$$
\int_{\Omega_{e}} W(\mathbf{F}) d V_{0} \approx \sum_{q=1}^{Q} \omega_{e}^{q} W\left(\mathbf{F}_{e}^{q}\right),
$$

where $Q$ is the order of the quadrature rule, $\omega_{e}^{q}$ are the quadrature weights for element $e$ and $\mathbf{F}_{e}^{q}$ is the deformation gradient evaluated at the quadrature point $q$ of element $e$. In the limit of linear triangular elements (as employed in paper II), a single quadrature point is used and the volume integral simply reduces to $\Omega_{e} \hat{W}\left(\mathbf{F}_{e}\right)$, where $\mathbf{F}_{e}$ is the constant deformation gradient in the element.

To find the equilibrium configuration we need to minimize the potential energy $\widetilde{\Pi}\left(\left\{\mathbf{u}_{i}\right\}\right)$ in Eq. (6). We can use either a conjugate gradient algorithm (see Ref. 8 for more detail) which requires the gradient of $\widetilde{\Pi}$ with respect to $\mathbf{u}_{a}$ or quasi-Newton schemes (see Ref. 9) which have superior convergence properties but also require the second derivative matrix or Hessian of Eq. (6). Differentiating Eq. (6) we have

$$
\frac{\partial \widetilde{\Pi}}{\partial u_{a i}}=\sum_{e=1}^{N_{e}} \int_{\Omega_{e}} P_{i J} N_{a, J} d V_{0}-\int_{\partial B_{0 T}} \bar{T}_{i} N_{a} d S_{0},
$$

where $\mathbf{P}$ is the first Piola-Kirchhoff stress tensor defined by

$$
P_{i J}=\frac{\partial W}{\partial F_{i J}} .
$$

Note that the first Piola-Kirchhoff stress is a mixed tensor, i.e., it has one index in the reference configuration and one in the spatial configuration and it is not symmetric.

The Hessian can similarly be obtained

$$
\frac{\partial^{2} \widetilde{\Pi}}{\partial u_{a i} \partial u_{b j}}=\sum_{e=1}^{N_{e}} \int_{\Omega_{e}}\left[D_{i J k L} N_{a, J} N_{b, L}\right] d V_{0},
$$

where $\mathbf{D}$ are the mixed tangential moduli,

$$
D_{i J k L}=\frac{\partial^{2} W}{\partial F_{i J} \partial F_{k L}} .
$$

We now have expressions for the gradient and Hessian of the potential energy in Eqs. (8) and (10) in terms of the stresses and elastic moduli within the elements. These depend on our choice of a strain energy density function $W$ through an appropriate atomistic model.

\section{ATOMISTIC CONSTITUTIVE LAW FOR COMPLEX BRAVAIS LATTICES}

In order to formulate a constitutive law for a continuum from the atomic interactions of the discrete crystal that underlies it, we must hypothesize a connection between the continuum displacement field and the motion of atoms. The standard reasoning consistent with the locality approximation of continuum mechanics is that the atomic environment at a continuum point is characterized by the deformation gradient there; this is referred to as the Cauchy-Born hypothesis. ${ }^{10}$ Thus, each continuum point is taken to represent a large, essentially infinite, region on the atomic scale which is homogeneously distorted according to the deformation gradient at the point. The energy of the distorted crystal and its derivatives can then be obtained from any atomistic model of choice from approximate classical descriptions to rigorous first-principles methods through the use of periodic boundary conditions.

It is worth noting the limitations of the local approximation. First, a local formulation has no internal length scale, thus it is the strain in an element and not its absolute size that enters into the constitutive calculation. This approximation will be valid as long as the continuum displacement field is slowly varying on the atomic scale. If it is not, a nonlocal formulation will be necessary (see Ref. 11 for details). Second, surfaces and other interfaces will be invisible to a local formulation. It is possible within a local formulation to include regions with different constitutive descriptions, e.g., as in a polycrystal, and of course in a numerical simulation the model is finite and must terminate at surfaces. However, the energetics of the interfaces will not be accounted for since, as stated, the constitutive calculations at each point are performed for an infinite crystal free of boundaries. Third, the details of atomic scale defects such as dislocation cores will not be correctly reproduced. Interestingly, though, due to the nonconvexity of an atomistically calculated strain energy function, a local model can sustain stable dislocations with finite core energies. This is because the energy is computed from the atomic interactions in a crystal and thus all symmetries are automatically reproduced and more importantly lattice invariant shears are recognized. As a result, when a lattice is sheared by a full Burgers vector, as it is in the wake of a dislocation, the energy penalty is nil and the dislocation is stable (see Ref. 1 for details). The relaxed core structure, however, will only be a crude approximation to the correct structure.

Now let us consider the application of the Cauchy-Born rule to complex Bravais lattices. We consider a crystal which may be represented as a Bravais lattice with Bravais vectors 
$\mathbf{A}_{k}$ and $\mathcal{N}_{a}+1$ basis atoms per Bravais site. The coordinates of the atoms making up the lattice in the reference coordinate system will be

$$
\mathbf{X}^{\nu}=\ell_{k}^{N} \mathbf{A}_{k}+\mathbf{b}^{n}
$$

where $N$ is the Bravais site number running from 0 to $\mathcal{N}_{s}$, if there are $\mathcal{N}_{s}+1$ sites, and 0 corresponds to the origin site (i.e., $\left.\ell_{k}^{0}=0\right), n$ is an index referring to the basis atom number, $\ell^{N}$ is a triplet of integers locating Bravais site $N$ in space and $\mathbf{b}^{n}$ is the position of basis atom $n$ relative to the Bravais site. In order to simplify the notation we bundle the site and basis atom number into a single index denoted by a Greek letter, e.g., $\nu=(N, n)$, which completely identifies an atom. Without loss of generality, we may set $\mathbf{b}^{0}=0$ so that one basis atom always lies at the Bravais site. Applying the Cauchy-Born rule, the positions of the atoms after deformation is given by

$$
\mathbf{x}^{\nu}=\mathbf{F}\left(\mathbf{X}^{\nu}+\zeta^{n}\right)=\mathbf{F}\left(\ell_{k}^{N} \mathbf{A}_{k}+\mathbf{b}^{n}+\boldsymbol{\zeta}^{n}\right),
$$

where $\zeta^{n}$ are additional inner displacements resulting from energy relaxation with respect to the basis atom positions (see Refs. 12-14 for details). Again, without loss of generality and in order to rule out rigid-body translation we may fix the lattice by setting $\zeta^{0}=0$. By defining the inner displacements $\boldsymbol{\zeta}^{n}$ in the reference configuration we automatically guarantee the invariance of these measures with respect to rigid-body rotation. ${ }^{12}$

In general, the energy of a collection of atoms $\left\{\mathbf{x}^{\nu}\right\}$ will be a function of their coordinates,

$$
E=E\left(\left\{\mathbf{x}^{\nu}\right\}\right) .
$$

The strain energy density $W$ follows by normalizing this quantity with respect to the atomic volume in the reference configuration $\Omega$,

$$
W=\frac{1}{\Omega} E\left(\left\{\mathbf{x}^{\nu}\right\}\right) .
$$

We stated earlier that the strain energy function must obey material frame indifference, i.e., invariance with respect to rigid-body rotation. Martin ${ }^{13}$ studied the implication of this to atomic energy functions and concluded that the energy can only depend on the atomic coordinates through scalar products of their relative positions. We define $\mathbf{R}$ and $\mathbf{r}$ as relative position vectors between pairs of atoms in the reference and deformed configurations, respectively,

$$
\mathbf{R}^{\nu \mu}=\mathbf{X}^{\mu}-\mathbf{X}^{\nu}=\left(\ell_{k}^{M}-\ell_{k}^{N}\right) \mathbf{A}_{k}+\mathbf{b}^{m}-\mathbf{b}^{n},
$$

here $\mu=(M, m)$ and

$$
\mathbf{r}^{\nu \mu}=\mathbf{x}^{\mu}-\mathbf{x}^{\nu}=\mathbf{F}\left[\left(\ell_{k}^{M}-\ell_{k}^{N}\right) \mathbf{A}_{k}+\mathbf{b}^{m}-\mathbf{b}^{n}+\boldsymbol{\zeta}^{m}-\boldsymbol{\zeta}^{n}\right] .
$$

From Eq. (17) it is clear that scalar dot products of $\mathbf{r}$ will depend on $\mathbf{F}$ through $\mathbf{F}^{T} \mathbf{F}$ or $\mathbf{C}$ as expected from material frame indifference. For example, the magnitude of $\mathbf{r}^{\nu \mu}$ will be given by

$$
r^{\nu \mu}=\left\{\left[\mathbf{X}^{\mu}-\mathbf{X}^{\nu}+\boldsymbol{\zeta}^{m}-\boldsymbol{\zeta}^{n}\right]^{T} \mathbf{F}^{T} \mathbf{F}\left[\mathbf{X}^{\mu}-\mathbf{X}^{\nu}+\boldsymbol{\zeta}^{m}-\boldsymbol{\zeta}^{n}\right]\right\}^{1 / 2}
$$

Other scalar products will have a similar dependence. Thus, in general, we have $W=W\left(\mathbf{F}, \zeta^{1}, \cdots, \boldsymbol{\zeta}^{N_{a}}\right)$. For a given macroscopic deformation, the strain energy must be minimized with respect to the inner displacements, ${ }^{14}$ thus we require

$$
\left.\frac{\partial W}{\partial \boldsymbol{\zeta}^{n}}\right|_{\mathbf{F}}=0, \quad n=1, \ldots, \mathcal{N}_{a},
$$

and $\partial^{2} W / \partial \boldsymbol{\zeta}^{m} \partial \zeta^{n}$ must be positive definite. The relations in Eq. (19) form a system of $3 \mathcal{N}_{a}$ equations for the $3 \mathcal{N}_{a}$ unknowns $\zeta_{J}^{n}$. The dependence of $W$ on $\zeta^{n}$ will generally be highly complex and the resulting system of equations coupled and nonlinear. Practically, the solution of Eq. (19) is best achieved by employing a numerical minimization algorithm, such as the conjugate gradient method, to minimize $W$ with respect to the inner displacements with the deformation gradient $\mathbf{F}$ held fixed. This minimization can be strongly history dependent at zero temperature (i.e., the local minimum to which it converged will depend on the initial guess). Thus, it is necessary to store the inner displacements $\boldsymbol{\zeta}^{n}$ at each quadrature point, using the previous solution at that point as the initial guess for the next iteration.

After minimization, we obtain the equilibrium inner displacements $\hat{\boldsymbol{\zeta}}^{n}=\hat{\boldsymbol{\zeta}}^{n}(\mathbf{F})$, which implies that the strain energy is only a function of $\mathbf{F}$,

$$
W=W\left(\mathbf{F}, \hat{\boldsymbol{\zeta}}^{n}\right)=W\left(\left(\mathbf{F}, \hat{\boldsymbol{\zeta}}^{n}(\mathbf{F})\right) \equiv \hat{W}(\mathbf{F})\right.
$$

We may now compute the stress and moduli tensors in the presence of internal relaxation. The first Piola-Kirchhoff stress was defined earlier in Eq. (9). For a complex Bravais lattice we have

$$
P_{i J}=\frac{\partial \hat{W}}{\partial F_{i J}}=\left.\frac{\partial W}{\partial F_{i J}}\right|_{\hat{\zeta}}+\left.\left.\frac{\partial W}{\partial \hat{\zeta}_{K}^{n}}\right|_{\hat{\zeta}} \frac{\partial \hat{\zeta}_{K}^{n}}{\partial F_{i J}}\right|_{\hat{\zeta}},
$$

where the subscript $\hat{\zeta}$ indicates the expression is evaluated at the relaxed inner displacements $\hat{\boldsymbol{\zeta}}^{n}$ and repeat indices on vector quantities ( $K$ and $n$ on $\zeta$ ) implies summation over all components. Under ideal conditions the second term in Eq. (21) drops out due to Eq. (19) and we are left with the standard definition for the stress tensor evaluated at the relaxed inner displacements

$$
\mathbf{P}=\left.\frac{\partial W}{\partial \mathbf{F}}\right|_{\hat{\zeta}} .
$$

However, since in practical applications Eq. (19) is only satisfied approximately to some given precision, it is preferable to work with the more complicated expression in Eq. (21). If Eq. (22) is used instead, the energy and its stress derivative would not be compatible and numerical difficulties will ensue at the final stages of convergence where the minimum of the energy surface would not correspond to a stress-free state. To use Eq. (21) we need to compute the nontrivial derivative $\partial \boldsymbol{\zeta}^{n} / \partial \mathbf{F}$. To this end we differentiate Eq. (19) with respect to $F_{k L}$ and obtain

$$
\frac{\partial^{2} W}{\partial \zeta_{I}^{m} \partial \zeta_{J}^{n}} \frac{\partial \zeta_{I}^{m}}{\partial F_{k L}}=-\frac{\partial^{2} W}{\partial F_{k L} \partial \zeta_{J}^{n}} .
$$


Inverting this relation we find the required derivative

$$
\frac{\partial \zeta_{I}^{m}}{\partial F_{k L}}=-\left[\frac{\partial^{2} W}{\partial \zeta_{I}^{m} \partial \zeta_{J}^{n}}\right]^{-1} \frac{\partial^{2} W}{\partial F_{k L} \partial \zeta_{J}^{n}}
$$

To obtain the mixed tangential moduli tensor $\mathbf{D}$ in Eq. (11) we must differentiate Eq. (21) with respect to F, explicitly accounting for the dependence of $\hat{\boldsymbol{\zeta}}^{n}$ on it. Differentiating, rearranging and simplifying by making use of Eq. (19), we find

$$
\begin{aligned}
D_{i J k L}= & \frac{\partial^{2} W}{\partial F_{i J} \partial F_{k L}}+\frac{\partial^{2} W}{\partial F_{i J} \partial \zeta_{P}^{m}} \frac{\partial \zeta_{P}^{m}}{\partial F_{k L}}+\frac{\partial^{2} W}{\partial F_{k L} \partial \zeta_{P}^{m}} \frac{\partial \zeta_{P}^{m}}{\partial F_{i J}} \\
& +\frac{\partial^{2} W}{\partial \zeta_{P}^{m} \partial \zeta_{Q}^{n}} \frac{\partial \zeta_{P}^{m}}{\partial F_{i J}} \frac{\partial \zeta_{Q}^{n}}{\partial F_{k L}},
\end{aligned}
$$

where for notational simplicity we have dropped the indication that all terms are evaluated at $\hat{\boldsymbol{\zeta}}^{n}$, but this is implied. The last two terms in Eq. (25) can be shown to cancel when Eq. (23) is substituted into the last term and we are left with the following simpler relation:

$$
D_{i J k L}=\frac{\partial^{2} W}{\partial F_{i J} \partial F_{k L}}+\frac{\partial^{2} W}{\partial F_{i J} \partial \zeta_{P}^{m}} \frac{\partial \zeta_{P}^{m}}{\partial F_{k L}} .
$$

Finally, by substituting Eq. (24) into Eq. (26) we can obtain a more symmetric expression for the elastic moduli

$$
D_{i J k L}=\left.\left\{\frac{\partial^{2} W}{\partial F_{i J} \partial F_{k L}}-\left[\frac{\partial^{2} W}{\partial \zeta_{P}^{m} \partial \zeta_{Q}^{n}}\right]^{-1} \frac{\partial^{2} W}{\partial F_{i J} \partial \zeta_{P}^{m}} \frac{\partial^{2} W}{\partial F_{k L} \partial \zeta_{Q}^{n}}\right\}\right|_{\hat{\zeta}} .
$$

It is worth noting that the correction due to internal relaxation will be non-zero and may be significant even for an undistorted crystal (i.e., $\mathbf{F}=\mathbf{I}$ ). Thus, internal relaxation may not be negligible even for infinitesimal straining in some cases; this is the case for silicon.

As in the case of the stresses, an argument may be made that due to numerical precision considerations the full expression for the elastic moduli which does not assume Eq. (19) (not given here) should be used in lieu of the simplified expression in Eq. (27). However, since the elastic moduli are only used to obtain search directions and are not used as a convergence criterion the discrepancy is less significant and Eq. (27) may be used.

\section{Two-body and three-body separable potentials}

To this point the derivation was general without reference to a particular energy function. We now assume that the energy of a collection of identical atoms may be expressed in the form of a two-body and three-body separable potential,

$$
E=\sum_{\eta}\left[\frac{1}{2} \sum_{\mu} V_{2}\left(r^{\eta \mu}\right)+\sum_{\mu<\nu} V_{3}\left(r^{\eta \mu}, r^{\eta \nu}, \cos \theta^{\eta \mu \nu}\right)\right],
$$

where $r^{\eta \mu}$ is the distance between atoms $\eta$ and $\mu$ and $\theta^{\eta \mu \nu}$ is the angle defined by the triplet of atoms $\eta, \mu$, and $\nu$. In Eq. (28) a sum over a Greek index implies a double summation over the Bravais sites and basis atoms associated with the index. In this case the indices $\eta, \mu$, and $\nu$ run over all Bravais sites and associated basis atoms in the solid. Note that in Eq. (28) we have assumed a trigonometric dependence of the energy on the angle $\theta$. This is normally the case and greatly simplifies the expressions derived later in Appen$\operatorname{dix}$ A.

For a homogeneously distorted crystal, the same basis atoms at different lattice sites will experience identical environments and thus the calculation of Eq. (28) may be limited to a single unit cell in order to obtain the correct energy density. For convenience we choose to compute the energy with respect to the origin Bravais site $o=(0, a)$,

$$
W=\frac{1}{\Omega} \widehat{\sum_{o}}\left[\frac{1}{2} \sum_{\mu} V_{2}\left(r^{o \mu}\right)+\sum_{\mu<\nu} V_{3}\left(r^{o \mu}, r^{o \nu}, \cos \theta^{o \mu \nu}\right)\right] \text {, }
$$

where $\Omega$ is the primitive Bravais cell volume, $r^{o \mu}$ is the distance of atom $\mu$ from basis atom $a$ of the origin Bravais site, and $\theta^{o \mu \nu}$ is the angle measured relative to that atom. The $\hat{\Sigma}$ symbol indicates summation only over the second component of a Greek index (i.e., over $a$ in this case).

Given the specific potential description of Eq. (29) we may explicitly obtain the derivatives of the strain energy density appearing in the stress (21) and moduli (27) expressions. The first derivatives are given

$$
\begin{aligned}
\frac{\partial W}{\partial F_{i J}}= & \frac{1}{\Omega} \sum_{o}\left\{\frac{1}{2} \sum_{\mu} V_{2, \mu} \frac{\partial r^{o \mu}}{\partial F_{i J}}+\sum_{\mu<\nu}\left[V_{3, \mu} \frac{\partial r^{o \mu}}{\partial F_{i J}}+V_{3, \nu} \frac{\partial r^{o \nu}}{\partial F_{i J}}\right.\right. \\
& \left.\left.+V_{3, \theta} \frac{\partial \cos \theta^{o \mu \nu}}{\partial F_{i J}}\right]\right\}, \\
\frac{\partial W}{\partial \zeta_{K}^{p}}= & \frac{1}{\Omega} \sum_{o}\left\{\frac{1}{2} \sum_{\mu} V_{2, \mu} \frac{\partial r^{o \mu}}{\partial \zeta_{K}^{p}}+\sum_{\mu<\nu}\left[V_{3, \mu} \frac{\partial r^{o \mu}}{\partial \zeta_{K}^{p}}+V_{3, \nu} \frac{\partial r^{o \nu}}{\partial \zeta_{K}^{p}}\right.\right. \\
& \left.\left.+V_{3, \theta} \frac{\partial \cos \theta^{o \mu \nu}}{\partial \zeta_{K}^{p}}\right]\right\} .
\end{aligned}
$$

In the above expressions ( $)_{, \mu}$ indicates differentiation with respect to $r^{o \mu}$ and ( ) ${ }_{\theta}$ indicates differentiation with respect to $\cos \theta^{\rho \mu \nu}$. The second derivatives can easily be obtained in similar fashion and are not given here for reasons of brevity. The derivatives in Eqs. (30) and (31) and the second derivatives contain partial derivatives of the form $\partial r / \partial \mathbf{F}, \partial r / \partial \boldsymbol{\zeta}$, $\ldots$. These are kinematic identities and they are given in Appendix A.

When the energy is computed classically as in Eq. (29), it is necessary to locate all neighboring atoms displaced to within the computed atom's cutoff radius upon deformation. However, obtaining an atom's neighbors in a deformed crystal is computationally intensive. A convenient approach, is to construct a crystal template. This is a list of the atomic coordinates of all atoms within some large radius of the unit cell sorted by distance from the basis atoms. It is then possible to compute a conservative estimate for the outer radius from which atoms could move inside the cutoff sphere. This is referred to as an influence radius. A derivation of this measure for complex Bravais lattices is given in Appendix C. 
In simulations, this influence radius serves two purposes. First, it is used to ensure that the crystal template stored for the energy calculations is sufficiently large. Second, the influence radius is used to identify the smallest subset of atoms which have a possibility of entering into the cutoff radius, thereby making the energy calculations as efficient as possible.

\section{Tight-binding models}

A completely different method for computing the energy of a collection of atoms uses the tight-binding method, a simple quantum-mechanical model. ${ }^{15}$ In this method the atoms are treated as classical particles that interact in part through an effective potential exerted by the electrons that are treated quantum mechanically. Hypothetical basis orbitals with the angular symmetries of single atom eigenstates are centered around each atom. The details of the basis functions do not enter into the energy calculation, but only the interactions between basis elements $\left|\phi_{i}\right\rangle$ that form the overlap matrix

$$
S_{i j}=\left\langle\phi_{i} \mid \phi_{j}\right\rangle,
$$

and the Hamiltonian matrix

$$
H_{i j}=\left\langle\phi_{i}|H| \phi_{j}\right\rangle .
$$

The indices $i$ and $j$ run over the all the atoms and all the basis elements centered on each atom. The energy eigenvalues are found by solving the generalized eigenvalue equation

$$
H_{i j} \psi_{n j}=\epsilon_{n} S_{i j} \psi_{n j},
$$

where $\epsilon_{n}$ is the $n$th eigenvalue and $\psi_{n j}$ is the $j$ th component of the $n$th eigenvector.

In the particular tight-binding model used here, the energy density, $W$, is made up of a classical two-body pair repulsion term and a band-structure term ${ }^{16}$

$$
W=\frac{1}{\Omega} \widehat{\sum_{o}}\left[\frac{1}{2} \sum_{\mu} V_{2}\left(r^{o \mu}\right)\right]+\frac{2}{\Omega N_{k}} \sum f_{n} \epsilon_{n},
$$

where $N_{k}$ is the number of $k$ points, and the sum $\Sigma_{n}$ is over both bands and $k$ points. The distribution $f_{n}$ is taken to be the Fermi function,

$$
f_{n}=\frac{1}{1+e^{\left[\left(\epsilon_{n}-\epsilon_{f}\right) / \sigma\right]}},
$$

where $\epsilon_{f}$ is the Fermi energy, and $\sigma$ is a smoothing parameter. The smoothing parameter is used to maintain numerical stability when configurations with no band gap are treated with a finite $k$ point mesh in the course of a simulation. It is not related to the physical temperature of the system.

Ideally simulation results should not depend on the value of $\sigma$. Da $\mathrm{Vita}^{17}$ has shown that while the energy and freeenergy density both vary as $\sigma^{2}$, the average of the two varies as $\sigma^{4}$ (see Appendix B). Thus for the tight-binding model we use an average of the energy and free-energy density, denoted $\widetilde{W}$, which has an entropic term added to the expression in Eq. (35),

$$
\widetilde{W}=W+\frac{\sigma}{\Omega N_{k}} \sum_{n}\left[f_{n} \ln \left(f_{n}\right)+\left(1-f_{n}\right) \ln \left(1-f_{n}\right)\right] .
$$

For the derivatives,

$$
\begin{aligned}
\frac{\partial \widetilde{W}}{\partial F_{i J}}= & \frac{1}{\Omega} \sum_{o}\left[\frac{1}{2} \sum_{\mu} V_{2, \mu} \frac{\partial r^{o \mu}}{F_{i J}}\right] \\
& +\frac{1}{\Omega N_{k}} \sum_{n}\left[2 f_{n}-\frac{1}{\sigma} \hat{f}_{n}\left(\epsilon_{n}-\frac{\sum_{m} \epsilon_{m} \hat{f}_{m}}{\sum_{m} \hat{f}_{m}}\right)\right] \frac{\partial \epsilon_{n}}{\partial F_{i J}}
\end{aligned}
$$

where

$$
\hat{f}_{n}=\frac{e^{\left[\left(\epsilon_{n}-\epsilon_{f}\right) / \sigma\right]}}{\left(1+e^{\left[\left(\epsilon_{n}-\epsilon_{f}\right) / \sigma\right]}\right)^{2}} .
$$

With the aid of the Hellman-Feynman theorem ${ }^{18,19}$ one can show,

$$
\frac{\partial \epsilon_{n}}{\partial F_{i J}}=\frac{1}{2} \sum_{o} \sum_{\mu}\left[\sum_{l m} \psi_{n l}^{*}\left(\frac{\partial H_{l m}}{\partial r_{i}^{o \mu}}-\epsilon^{n} \frac{\partial S_{l m}}{\partial r_{i}^{o \mu}}\right) r_{k}^{o \mu} \psi_{n m}\right] F_{J k}^{-1},
$$

where $r_{i}^{o \mu}$ is the $i$ th component of the the position vector between the atoms $o$ and $\mu$.

\section{SIGNIFICANCE OF INTERNAL RELAXATION}

So far the derivation has been given in general terms for arbitrary crystal structures. To demonstrate the benefits and implications of implementing an atomistically based constitutive law we turn to the specific example of silicon. In particular, we will investigate the effect of internal atomic relaxation on observable macroscopic response. We compare two different atomistic descriptions, the classical StillingerWeber (SW) model ${ }^{20}$ and a nonorthogonal tight-binding Hamiltonian (TB) developed by Bernstein and Kaxiras. ${ }^{16}$

We focus on a simple shear deformation. We choose a [110] shear direction with a [111] slip plane normal. The corresponding deformation gradient has the form

$$
\mathbf{F}=\mathbf{I}+\gamma \mathbf{S} \otimes \mathbf{n}
$$

where $\gamma$ is the shear parameter, $\mathbf{s}$ is the slip direction, and $\mathbf{n}$ is the slip plane normal. Figure 3 presents the strain energy density of the crystal as it is sheared. In frame (a) no internal relaxation is allowed, while in frame (b) relaxation is taken into account. The difference in the energies are startling. Allowing the second basis atom to relax reduces the maximum energy by a factor of nearly 30. This is emphasized in the figure by the dashed inset in frame (a) which corresponds to the domain of frame (b).

In addition to the dramatic reduction in energy, and perhaps more importantly, the periodic nature of the response is also affected by relaxation. Whereas in the unrelaxed case the perfect crystal structure is restored after shearing by $\gamma$ $=2 \sqrt{6}$, when the second basis atom is allowed to relax, the period is reduced by a factor of four, i.e., $\gamma=\sqrt{6} / 2$. The 


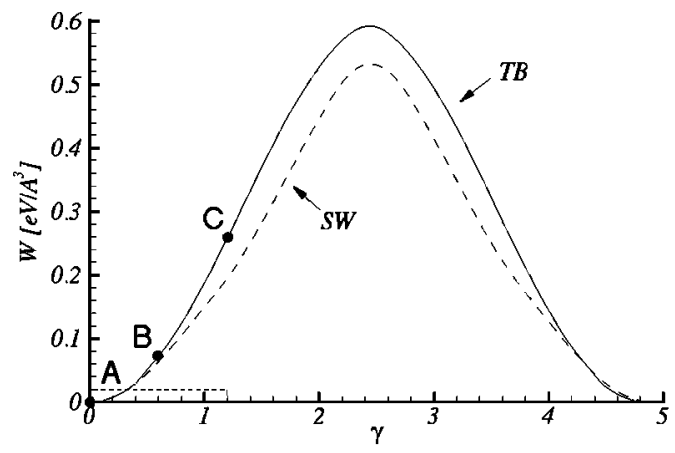

(a)

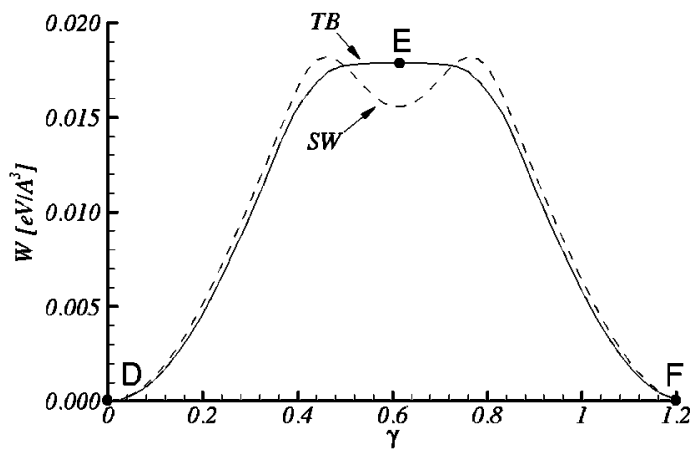

(b)

FIG. 3. Strain energy density of a silicon crystal as it is sheared along the $[\overline{1} 10] /(111)$ slip system. Frame (a) presents the case where no internal relaxation is allowed. In frame (b), which occupies the dashed inset in (a), relaxation is taken into account. Solid lines were computed using the TB formalism, dashed lines with the SW potential.

reason for this becomes apparent by studying a single tetrahedron as it is sheared with and without internal relaxation as seen in Fig. 4. The lighter atoms are the Bravais site atoms that are constrained to move according to the applied deformation field. The dark atom is the second basis atom which in frame (a) is constrained along with the Bravais site atoms and in (b) is free to move relative to them. Each frame contains a series of three snapshots labeled by letters as indicated in Fig. 3.

In the unrelaxed case, we see that the second basis atom lags further and further behind the triplet of atoms above it. The tetrahedron gets more and more distorted as the crystal is sheared. At point $\mathrm{C}$ the crystal structure is far from the ideal diamond structure with a correspondingly high energy. The perfect structure will be restored only by shearing four times further. When relaxation is taken into account the second basis atom displaces to minimize the distortion. The initial structure in $D$ is, of course, the same as $A$ in the unrelaxed case, but then as the upper triangle moves to the right, the second basis atom moves with it, remaining underneath. In this manner, the bond angles between the second basis
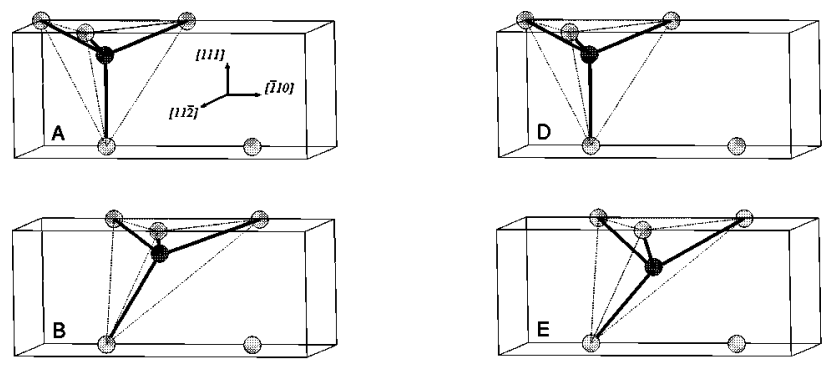

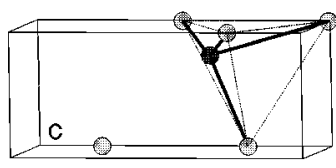

(a)

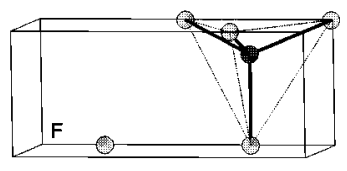

(b)
FIG. 4. Shearing of a single silicon tetrahedron (a) without internal relaxation and (b) with internal relaxation. The dark atom is the second basis atom which in frame (b) is allowed to relax at each step. The letters correspond to points on the graphs in Fig. 3. All structures were computed using the TB formalism. atom and the upper triplet of atoms, remain closer to the ideal $109.47^{\circ}$. As the shear progresses, the lower left atom falls further behind causing the second basis atom to move down in the $[\overline{1} \overline{1} \overline{1}]$ direction and slightly back in the [1 $\overline{1} 0]$ direction to reduce the strain on that bond (see snapshot $\mathrm{E}$ ). As the lower right atom draws closer, the second basis atom is pulled towards it, now moving a little ahead of the upper triangle, and eventually the perfect crystal structure is restored in $\mathrm{F}$.

Finally, it is of interest to compare the predictions of the tight-binding model with the Stillinger-Weber potential. The tight-binding approach is attractive because its quantummechanics basis promises for a more transferable model, however it is computationally far more intensive than classical approaches [e.g., computing the relaxed curves in Fig. 3(b) takes 300 times longer using the TB method than with the SW potential]. For the simple shear deformation studied here both approaches yield rather similar results. The overall energetics are close and the structures observed along the shear trajectory were nearly identical. The main concern associated with the SW potential is the appearance of a nonphysical local minimum corresponding to the midshear position [see Fig. 3(b)]. This appears to be a general feature of classical potentials. In fact, it appears that the more elaborate the potential and the more it is fitted to given structures, the more undesirable local minima appear between these desired states. This may not be of concern in finite temperature simulations where the system has sufficient thermal energy to escape the local wells, however at zero temperature these minima can be problematic. Thus, in addition to transferability, the smoothness of the energy surface of more rigorous quantum-mechanical approaches is of significant benefit. These issues will be pursued in more detail in a companion paper where more complex deformation pathways will be studied. $^{2}$

\section{SUMMARY AND CONCLUSIONS}

We have formulated a finite element method for materials with multiple atoms in a unit cell that uses atomistic energy functionals to determine the constitutive relations. The method presented here naturally incorporates crystal anisot- 
ropy, nonlinear contributions to the energy density, and the possibility of structural phase transitions within the finite element framework. We have derived explicit expressions for an empirical potential with two- and three-body terms, and for a two-center tight-binding Hamiltonian. Throughout, special care has be taken to correctly treat the internal degrees of freedom. We have shown that correct treatment of these degrees of freedom dramatically affects the behavior of even a simple system such as sheared bulk silicon.

\section{ACKNOWLEDGMENTS}

This work was supported by Harvard's Materials Science and Engineering Center, which is funded by the NSF, Grant No. DMR-94-00396. The authors gratefully acknowledge helpful discussions with M. Z. Bazant, R. Miller, R. Phillips, and U. V. Waghmare.

\section{APPENDIX A: DERIVATIVES OF THE KINEMATICAL MEASURES}

In this section we give the final expressions for the first and second derivatives of $r^{o \mu}$, the distance between atom $\mu$ and an atom at the origin, and $\cos \theta^{\rho \mu \nu}$, the angle between two atoms $\mu$ and $\nu$ and the origin, with respect to the deformation gradient and internal displacements. We follow the notation of Sec. III, i.e., $r^{o \mu}$ is defined according to Eq. (18), $\cos \theta^{o \mu \nu}$ is given by $\cos \theta^{\rho \mu \nu}=\left(\mathbf{r}^{o \mu} \cdot \mathbf{r}^{o \nu}\right) / r^{o \mu} r^{o \nu}$, and $\boldsymbol{\rho}$ is the unit vector in the direction of $\mathbf{r}, \rho_{i}^{o \mu}=r_{i}^{o \mu} / r^{o \mu}$.

The first derivatives are given by

$$
\begin{gathered}
\frac{\partial r^{o \mu}}{\partial F_{i J}}=r^{o \mu}\left[\rho_{i}^{o \mu} \rho_{j}^{o \mu}\right] F_{J j}^{-1}, \\
\frac{\partial r^{o \mu}}{\partial \zeta_{K}^{p}}=\left(\delta_{m p}-\delta_{a p}\right) \rho_{k}^{o \mu} F_{k K},
\end{gathered}
$$

$$
\begin{aligned}
\frac{\partial \cos \theta^{o \mu \nu}}{\partial F_{i J}}= & {\left[\rho_{i}^{o \mu} \rho_{j}^{o \nu}+\rho_{i}^{o \nu} \rho_{j}^{o \mu}\right.} \\
& \left.-\cos \theta^{o \mu \nu}\left(\rho_{i}^{o \mu} \rho_{j}^{o \mu}+\rho_{i}^{o \nu} \rho_{j}^{o \nu}\right)\right] F_{J j}^{-1},
\end{aligned}
$$

$$
\begin{aligned}
\frac{\partial \cos \theta^{o \mu \nu}}{\partial \zeta_{K}^{p}}= & {\left[\frac{\delta_{m p}-\delta_{a p}}{r^{o \mu}}\left(\rho_{k}^{o \nu}-\rho_{k}^{o \mu} \cos \theta^{o \mu \nu}\right)+\frac{\delta_{n p}-\delta_{a p}}{r^{o \nu}}\right.} \\
& \left.\times\left(\rho_{k}^{o \mu}-\rho_{k}^{o \nu} \cos \theta^{o \mu \nu}\right)\right] F_{k K}
\end{aligned}
$$

The second derivatives are given by

$$
\begin{gathered}
\frac{\partial^{2} r^{o \mu}}{\partial F_{i J} \partial F_{k L}}=r^{o \mu}\left[\delta_{i k}-\rho_{i}^{o \mu} \rho_{k}^{o \mu}\right] \rho_{j}^{o \mu} \rho_{l}^{o \mu} F_{J j}^{-1} F_{L l}^{-1}, \\
\frac{\partial^{2} r^{o \mu}}{\partial F_{i J} \partial \zeta_{K}^{p}}=\left(\delta_{m p}-\delta_{a p}\right)\left[\delta_{i k} \rho_{j}^{o \mu}+\delta_{j k} \rho_{i}^{o \mu}-\rho_{i}^{o \mu} \rho_{j}^{o \mu} \rho_{k}^{o \mu}\right] F_{J j}^{-1} F_{k K}, \\
\frac{\partial^{2} r^{o \mu}}{\partial \zeta_{K}^{p} \partial \zeta_{L}^{q}}=\frac{\left(\delta_{m p}-\delta_{a p}\right)\left(\delta_{m q}-\delta_{a q}\right)}{r^{o \mu}}\left[\delta_{k l}-\rho_{k}^{o \mu} \rho_{l}^{o \mu}\right] F_{k K} F_{l L},
\end{gathered}
$$

$$
\begin{aligned}
\frac{\partial^{2} \cos \theta^{o \mu \nu}}{\partial F_{i J} \partial F_{k L}}= & \left\{\delta_{i k}\left[\rho_{j}^{o \mu} \rho_{l}^{o \nu}+\rho_{j}^{o \nu} \rho_{l}^{o \mu}-\cos \theta^{o \mu \nu}\left(\rho_{j}^{o \mu} \rho_{l}^{o \mu}+\rho_{j}^{o \nu} \rho_{l}^{o \nu}\right)\right]-\left(\rho_{i}^{o \mu} \rho_{j}^{o \nu}+\rho_{i}^{o \nu} \rho_{j}^{o \mu}\right)\left(\rho_{k}^{o \mu} \rho_{l}^{o \mu}+\rho_{k}^{o \nu} \rho_{l}^{o \nu}\right)-\left(\rho_{i}^{o \mu} \rho_{j}^{o \mu}+\rho_{i}^{o \nu} \rho_{j}^{o \nu}\right)\right. \\
& \left.\times\left(\rho_{k}^{o \mu} \rho_{l}^{o \nu}+\rho_{k}^{o \nu} \rho_{l}^{o \mu}\right)+\cos \theta^{o \mu \nu}\left[\rho_{i}^{o \mu} \rho_{j}^{o \mu} \rho_{k}^{o \nu} \rho_{l}^{o \nu}+\rho_{i}^{o \nu} \rho_{j}^{o \nu} \rho_{k}^{o \mu} \rho_{l}^{o \mu}+3 \rho_{i}^{o \mu} \rho_{j}^{o \mu} \rho_{k}^{o \mu} \rho_{l}^{o \mu}+3 \rho_{i}^{o \nu} \rho_{j}^{o \nu} \rho_{k}^{o \nu} \rho_{l}^{o \nu}\right]\right\} F_{J j}^{-1} F_{L l}^{-1},
\end{aligned}
$$

$$
\begin{aligned}
\frac{\partial^{2} \cos \theta^{o \mu \nu}}{\partial F_{i J} \partial \zeta_{K}^{p}}= & \left\{\frac { \delta _ { m p } - \delta _ { a p } } { r ^ { o \mu } } \left[\delta_{i k} \rho_{j}^{o \nu}+\delta_{j k} \rho_{i}^{o \nu}-\left(\rho_{i}^{o \mu} \rho_{j}^{o \nu}+\rho_{i}^{o \nu} \rho_{j}^{o \mu}\right) \rho_{k}^{o \mu}-\left(\rho_{i}^{o \mu} \rho_{j}^{o \mu}+\rho_{i}^{o \nu} \rho_{j}^{o \nu}\right) \rho_{k}^{o \nu}-\cos \theta^{o \mu \nu}\left(\delta_{i k} \rho_{j}^{o \mu}+\delta_{j k} \rho_{i}^{o \mu}\right.\right.\right. \\
& \left.\left.-\left(\rho_{i}^{o \nu} \rho_{j}^{o \nu}+3 \rho_{i}^{o \mu} \rho_{j}^{o \mu}\right) \rho_{k}^{o \mu}\right)\right]+\frac{\delta_{n p}-\delta_{a p}}{r^{o \nu}}\left[\delta_{i k} \rho_{j}^{o \mu}+\delta_{j k} \rho_{i}^{o \mu}-\left(\rho_{i}^{o \mu} \rho_{j}^{o \nu}+\rho_{i}^{o \nu} \rho_{j}^{o \mu}\right) \rho_{k}^{o \nu}-\left(\rho_{i}^{o \mu} \rho_{j}^{o \mu}+\rho_{i}^{o \nu} \rho_{j}^{o \nu}\right) \rho_{k}^{o \mu}\right. \\
& \left.\left.-\cos \theta^{o \mu \nu}\left(\delta_{i k} \rho_{j}^{o \nu}+\delta_{j k} \rho_{i}^{o \nu}-\left(\rho_{i}^{o \mu} \rho_{j}^{o \mu}+3 \rho_{i}^{o \nu} \rho_{j}^{o \nu}\right) \rho_{k}^{o \nu}\right)\right]\right\} F_{J j}^{-1} F_{k K},
\end{aligned}
$$




$$
\begin{aligned}
\frac{\partial^{2} \cos \theta^{o \mu \nu}}{\partial \zeta_{K}^{p} \partial \zeta_{L}^{q}}= & \left\{\frac{\left(\delta_{m p}-\delta_{a p}\right)\left(\delta_{m q}-\delta_{a q}\right)}{\left(r^{o \mu}\right)^{2}}\left[-\rho_{k}^{o \mu} \rho_{l}^{o \nu}-\rho_{k}^{o \nu} \rho_{l}^{o \mu}+\cos \theta^{o \mu \nu}\left(3 \rho_{k}^{o \mu} \rho_{l}^{o \mu}-\delta_{k l}\right)\right]+\frac{\left(\delta_{n p}-\delta_{a p}\right)\left(\delta_{n q}-\delta_{a q}\right)}{\left(r^{o \nu}\right)^{2}}\right. \\
& \times\left[-\rho_{k}^{o \mu} \rho_{l}^{o \nu}-\rho_{k}^{o \nu} \rho_{l}^{o \mu}+\cos \theta^{o \mu \nu}\left(3 \rho_{k}^{o \nu} \rho_{l}^{o \nu}-\delta_{k l}\right)\right]+\frac{\left(\delta_{m p}-\delta_{a p}\right)\left(\delta_{n q}-\delta_{a q}\right)}{r^{o \mu} r^{o \nu}} \\
& \times\left[\delta_{k l}-\rho_{k}^{o \mu} \rho_{l}^{o \mu}-\rho_{k}^{o \nu} \rho_{l}^{o \nu}+\cos \theta^{o \mu \nu}\left(\rho_{k}^{o \mu} \rho_{l}^{o \nu}\right)\right]+\frac{\left(\delta_{n p}-\delta_{a p}\right)\left(\delta_{m q}-\delta_{a q}\right)}{r^{o \mu} r^{o \nu}} \\
& \left.\times\left[\delta_{k l}-\rho_{k}^{o \mu} \rho_{l}^{o \mu}-\rho_{k}^{o \nu} \rho_{l}^{o \nu}+\cos \theta^{o \mu \nu}\left(\rho_{k}^{o \nu} \rho_{l}^{o \mu}\right)\right]\right\} F_{k K} F_{l L} .
\end{aligned}
$$

\section{APPENDIX B: AVERAGED ENERGY DEPENDENCE ON SMOOTHING PARAMETER}

In this section we show that in a tight-binding-model the average of the energy and free-energy density $\widetilde{W}$ and the corresponding forces vary as $\sigma^{4}$. We assume that we have a smooth, continuous density of states, $g(\epsilon)$. Thus our starting expression for $\widetilde{W}$ is the same as the expression in Eq. (37), except that $\left(2 / \Omega N_{k}\right) \Sigma_{n}$ is replaced by $\int g(\boldsymbol{\epsilon}) d \epsilon$. We consider $\widetilde{W}$ to be a function of $\sigma$ and $\epsilon_{f}$, and require that the number density of electrons $N$ be independent of the smoothing parameter. Then,

$$
\frac{d \widetilde{W}}{d \sigma}=\frac{\partial \widetilde{W}}{\partial \sigma}-\frac{\partial \widetilde{W}}{\partial \epsilon_{f}}\left(\frac{\partial N}{\partial \sigma} / \frac{\partial N}{\partial \epsilon_{f}}\right)
$$

Determining the zero-order $\sigma$ contributions to the derivatives with respect to the Fermi energy is immediate, and additional terms in the expansion are of order $\sigma^{2}$,

$$
\begin{gathered}
\frac{\partial \widetilde{W}}{\partial \epsilon_{f}}=\epsilon_{f} g\left(\epsilon_{f}\right)+O\left(\sigma^{2}\right), \\
\frac{\partial N}{\partial \epsilon_{f}}=g\left(\epsilon_{f}\right)+O\left(\sigma^{2}\right) .
\end{gathered}
$$

The derivatives with respect to $\sigma$ can be evaluated using Sommerfield's lemma ${ }^{21}$

$$
\begin{gathered}
\frac{\partial \widetilde{W}}{\partial \sigma}=\frac{\pi^{2}}{3} \epsilon_{f} g^{\prime}\left(\epsilon_{f}\right) \sigma+O\left(\sigma^{3}\right), \\
\frac{\partial N}{\partial \sigma}=\frac{\pi^{2}}{3} g^{\prime}\left(\epsilon_{f}\right) \sigma+O\left(\sigma^{3}\right) .
\end{gathered}
$$

Combining Eqs. (B1)-(B5), we obtain

$$
\frac{d \widetilde{W}}{d \sigma} \approx O\left(\sigma^{3}\right)
$$

Taking the derivative of the expansion of $\widetilde{W}$ with respect to atom positions confirms that the forces have the same temperature dependence as $\widetilde{W}$.
In numerical tests of typical highly compressed silicon unit cells, we find that $\widetilde{W}$ is significantly less sensitive to the smoothing parameter value than the energy or free energy alone. Up to values of a few tenths of an $\mathrm{eV}$ for $\sigma, \widetilde{W}$ deviates from the zero-temperature energy by about $0.001 \%$, while the energy and free energy deviate by about $0.01 \%$. However, the behavior of the forces is worse by at least an order of magnitude. We keep the temperature as low as possible during simulations, between $10^{-2}$ and $10^{-3} \mathrm{eV}$.

\section{APPENDIX C: INFLUENCE RADIUS FOR COMPLEX LATTICES}

The energy and derivative computations in the main body of the paper are computationally intensive. It is of great practical importance to minimize the number of atoms that are involved in the computation. To this end we compute an influence radius. ${ }^{1}$ This is the distance of the furthest atom from the origin that will come within the cutoff radii of the computed Bravais site atoms. We obtain a continuum estimate for this measure.

Following the notation of Sec. III, a generic point $\mathbf{X}$ in the reference configuration is mapped to the deformed configuration by

$$
\mathbf{X}=\mathbf{F X}+\mathbf{z}
$$

where $\mathbf{z}=\mathbf{F} \boldsymbol{\zeta}$ is the inner relaxation in the deformed configuration. We wish to maximize $\mathbf{X}^{T} \mathbf{X}$ subject to the constraint $\mathbf{x}^{T} \mathbf{x}=r_{c}^{2}$, where $r_{c}$ is the cutoff radius. We define the functional

$$
\Lambda=\mathbf{X}^{T} \mathbf{X}-\mu\left(\mathbf{x}^{T} \mathbf{x}-r_{c}^{2}\right),
$$

where $\mu$ is a Lagrange multiplier. Inverting Eq. (C1) and substituting into Eq. (C2), we have

$$
\Lambda=\left(\mathbf{x}^{T}-\mathbf{z}^{T}\right) \mathbf{B}^{-1}(\mathbf{x}-\mathbf{z})-\mu\left(\mathbf{x}^{T} \mathbf{x}-r_{c}^{2}\right),
$$

where $\mathbf{B}^{-1}=\mathbf{F}^{-T} \mathbf{F}^{-1}$ is the inverse of the left Cauchy-Green deformation tensor. Taking a variation of Eq. (C3) with respect to $\mathbf{x}, \delta \Lambda=0$, leads to the system of equations

$$
\left(\mathbf{B}^{-1}-\mu \mathbf{I}\right) \mathbf{x}=\mathbf{B}^{-1} \mathbf{z} .
$$

In the absence of internal atoms, i.e., $\mathbf{z}=0$, this is a simple eigenvalue problem with the solution $R_{\mathrm{inf}}=r_{c} \sqrt{\mu_{\text {max }}}$, where 
$\mu_{\max }$ is the maximum eigenvalue of $\mathbf{B}^{-1}$. When $\mathbf{z}$ is not zero, Eq. (C4) is no longer an eigenvalue problem. Instead we need to solve for the value of $\mu$ by solving Eq. (C4), subject to the constraint $\mathbf{x}^{T} \mathbf{x}=r_{c}^{2}$. To facilitate this, we introduce the eigenvalues and eigenvectors of matrix $\mathbf{B}^{-1}, \lambda_{i}$ and $\boldsymbol{\xi}_{i}$, respectively, and express $\mathbf{x}$ and $\mathbf{B}^{-1} \mathbf{z}$ in this basis

$$
\begin{gathered}
\mathbf{x}=\sum_{i=1}^{3} \alpha_{i} \boldsymbol{\xi}_{i}, \\
\mathbf{B}^{-1} \mathbf{z}=\sum_{i=1}^{3} \beta_{i} \xi_{i} .
\end{gathered}
$$

Substituting Eqs. (C5) and (C6) into the system (C4) and making use of the eigenvalue identity,

$$
\mathbf{B}^{-1} \boldsymbol{\xi}_{i}=\lambda_{i} \boldsymbol{\xi}_{i}, \quad(\text { no sum })
$$

we obtain

$$
\sum_{i=1}^{3} \alpha_{i}\left(\lambda_{i}-\mu\right) \boldsymbol{\xi}_{i}=\sum_{i=1}^{3} \beta_{i} \xi_{i}
$$

We can then solve for $\alpha_{i}$

$$
\alpha_{i}=\frac{\beta_{i}}{\lambda_{i}-\mu}
$$

and thus obtain $\mathbf{x}$ from Eq. (C5),

$$
\mathbf{x}=\sum_{i=1}^{3} \frac{\beta_{i}}{\lambda_{i}-\mu} \boldsymbol{\xi}_{i}
$$

The coefficients $\beta_{i}$ in Eq. (C10) can readily be obtained from Eq. (C6). Applying $\boldsymbol{\xi}_{j}$ to both sides of Eq. (C6), making use of the symmetry of $\mathbf{B}^{-1}$ and the orthogonality of its eigenvectors and recalling relation $(\mathrm{C} 7)$, we find

$$
\beta_{i}=\lambda_{i}\left(\mathbf{z} \cdot \boldsymbol{\xi}_{i}\right)(\text { no sum }) .
$$

The remaining unknowns in Eq. (C10) are the values of the Lagrange multiplier $\mu$. We obtain an implicit equation for the values of $\mu$ by enforcing the constraint $\mathbf{x}^{T} \mathbf{x}=r_{c}^{2}$,

$$
\sum_{i=1}^{3} \frac{\lambda_{i}^{2}\left(\mathbf{z} \cdot \boldsymbol{\xi}_{i}\right)^{2}}{\left(\lambda_{i}-\mu\right)^{2}}=r_{c}^{2}
$$

where we have again made use of the orthogonality of the eigenvectors $\boldsymbol{\xi}_{i}$.

Equation (C12) may have as many as six distinct roots. These will appear in pairs around the eigenvalues of $\mathbf{B}^{-1}, \lambda_{i}$, where the left-hand side of Eq. (C12) is infinite. This can be seen in Fig. 5 where a typical curve for silicon is drawn.

Once the roots $\mu_{k}$ are found, we may compute the corresponding influence radii $R_{\mathrm{inf}}^{k}$,

$$
\left(R_{\text {inf }}^{k}\right)^{2}=\mathbf{X}^{T} \mathbf{X}=\left(\mathbf{x}_{k}^{T}-\mathbf{z}^{T}\right) \mathbf{B}^{-1}\left(\mathbf{x}_{k}-\mathbf{z}\right),
$$

where $\mathbf{x}_{k}$ is the solution (C10) with $\mu=\mu_{k}$. After substituting in the appropriate expressions and following some algebra we obtain

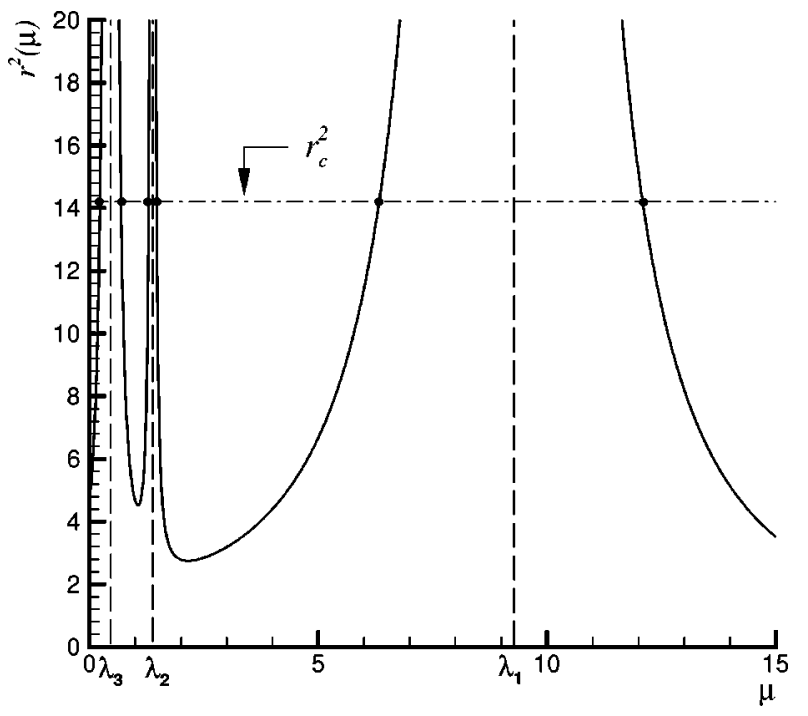

FIG. 5. A typical solution for the values of the Lagrange multiplier $\mu$. The curve is drawn for Stillinger-Weber silicon $\left(r_{c}=3.77\right.$ $\AA$ ) for a randomly selected deformation gradient and inner displacement. The six roots $\mu_{k}$ are indicated by black dots.

$$
\left(R_{\text {inf }}^{k}\right)^{2}=\mu_{k}^{2} \sum_{j=1}^{3} \frac{\lambda_{j}}{\left(\lambda_{j}-\mu_{k}\right)^{2}}\left(\mathbf{z} \cdot \boldsymbol{\xi}_{j}\right)^{2} .
$$

The influence radius $R_{\text {inf }}$ follows as

$$
R_{\mathrm{inf}}=\max _{k}\left\{R_{\mathrm{inf}}^{k}\right\}
$$

Equations (C12) and (C14) above represent a complete solution to the problem. However, solving for the roots $\mu_{k}$ and obtaining the eigenvalues and eigenvectors of $\mathbf{B}^{-1}$ may prove to be too time consuming for this to be worthwhile. It is thus of interest to obtain an approximate solution to the problem that will not underestimate the exact influence radius, and will be significantly faster.

Empirically, we observe that the maximum influence radius is always associated with the maximum $\mu$ root, i.e., the one to the right of the maximum eigenvalue $\lambda_{1}$ (see Fig. 5). We can approximately compute $\mu_{\max }$ by assuming that the term associated with $\lambda_{1}$ in Eq. (C12) dominates [since the denominator $\left(\lambda_{1}-\mu_{\max }\right)$ will be very small], thus

$$
\mu_{\max } \simeq \lambda_{1}+\frac{\lambda_{1}\left(\mathbf{z} \cdot \boldsymbol{\xi}_{1}\right)}{r_{c}}=\lambda_{1}\left[1+\frac{\mathbf{z} \cdot \boldsymbol{\xi}_{1}}{r_{c}}\right]
$$

Substituting Eq. (C16) into Eq. (C14), we have

$$
R_{\mathrm{inf}}^{2} \simeq \lambda_{1}^{2}\left[1+\frac{\mathbf{z} \cdot \boldsymbol{\xi}_{1}}{r_{c}}\right]^{2} \sum_{j=1}^{3} \frac{\lambda_{j}}{\left[\lambda_{1}\left(\mathbf{z} \cdot \boldsymbol{\xi}_{1}\right) / r_{c}+\lambda_{1}-\lambda_{j}\right]^{2}}\left(\mathbf{z} \cdot \boldsymbol{\xi}_{1}\right)^{2}
$$

The term $\mathbf{z} \cdot \boldsymbol{\xi}_{1}$ is bounded by $z=|\mathbf{z}|$, since $\boldsymbol{\xi}_{1}$ is a unit vector. In addition we may assume the $j=1$ term in Eq. (C17) dominates since the denominator is smallest and numerator greater there. Applying these assumptions and rearranging we find the final simplified estimate for the influence radius 


$$
R_{\mathrm{inf}}=\sqrt{\lambda_{1}} r_{c}\left(1+\frac{z}{r_{c}}\right)
$$

Interestingly, this expression is simply $\left(1+z / r_{c}\right) R_{\text {inf }}^{0}$, where

*Present address: Faculty of Mechanical Engineering, TechnionIsrael Institute of Technology, 32000 Haifa, Israel.

†Present address: Naval Research Lab, Washington, DC 20375.

${ }^{1}$ E. B. Tadmor, M. Ortiz, and R. Phillips, Philos. Mag. A 73, 1529 (1996).

${ }^{2}$ G. S. Smith, E. B. Tadmor, N. Bernstein, and E. Kaxiras (unpublished).

${ }^{3}$ E. B. Tadmor, U. V. Waghmare, and E. Kaxiras (unpublished).

${ }^{4}$ G. M. Pharr, W. C. Oliver, and D. S. Harding, J. Mater. Res. 6, 1129 (1991).

${ }^{5}$ D. Schryvers, Habilitation, University of Brussels, 1991.

${ }^{6}$ J. E. Marsden and T. J. R. Hughes, Mathematical Foundations of Elasticity (Dover, New York, 1983), Chap. 3.

${ }^{7}$ T. J. R. Hughes, The Finite Element Method (Prentice-Hall, Englewood Cliffs, NJ, 1987).

${ }^{8}$ M. Papadrakakis and P. Ghionis, Comput. Methods Appl. Mech. Eng. 59, 11 (1986).

${ }^{9}$ J. E. Dennis, Jr. and R. B. Schnabel, Numerical Methods for
$R_{\mathrm{inf}}^{0}$ is the influence radius for the shuffle-free case. Although, we have not established it rigorously, in practice this estimate has always behaved as a conservative bound on the actual influence radius.
Unconstrained Optimization and Nonlinear Equations (PrenticeHall, Englewood Cliffs, NJ, 1983).

${ }^{10}$ F. Milstein, in Mechanics of Solids, edited by H. G. Hopkins and M. J. Sewell (Pergamon Press, Oxford, 1982).

${ }^{11}$ V. B. Shenoy, R. Miller, E. B. Tadmor, D. Rodney, Rob Phillips, and M. Ortiz, J. Mech. Phys. Solids (to be published).

${ }^{12}$ J. W. Martin, J. Phys. C 8, 2858 (1975).

${ }^{13}$ J. W. Martin, J. Phys. C 8, 2837 (1975).

${ }^{14}$ C. S. G. Cousins, J. Phys. C 11, 4867 (1978).

${ }^{15}$ W. A. Harrison, Electronic Structure and the Properties of Solids (Freeman, San Francisco, 1980).

${ }^{16}$ N. Bernstein and E. Kaxiras, Phys. Rev. B 56, 10488 (1997).

${ }^{17}$ A. Da Vita, Ph.D. thesis, Keele University, 1992.

${ }^{18}$ H. Hellman, Einfrüng in die Quantenchemie (Deuticke, Leipzig, 1937).

${ }^{19}$ R. P. Feynman, Phys. Rev. 56, 340 (1939).

${ }^{20}$ F. H. Stillinger and T. A. Weber, Phys. Rev. B 31, 5262 (1985).

${ }^{21}$ A. Z. Sommerfeld, Z. Phys. 47, 1 (1928). 